\title{
Carnets
}

Revue électronique d'études françaises de l'APEF

Deuxième série - 8 | 2016

Du Français en cause aux causes du français

\section{Le français au Portugal}

De la langue si peu « Étrangère » à la difficile normalisation.

Considérations et perspectives

José Domingues de Almeida

\section{(2) OpenEdition}

1 Journals

Édition électronique

URL : http://journals.openedition.org/carnets/1891

DOI : 10.4000/carnets.1891

ISSN : 1646-7698

Éditeur

APEF

Référence électronique

José Domingues de Almeida, «Le français au Portugal », Carnets [En ligne], Deuxième série - 8| 2016, mis en ligne le 30 novembre 2016, consulté le 21 avril 2019. URL : http://journals.openedition.org/ carnets/1891; DOI : 10.4000/carnets.1891

Ce document a été généré automatiquement le 21 avril 2019

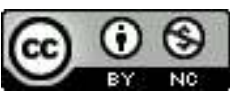

Carnets est mis à disposition selon les termes de la licence Creative Commons - Atribution - Pas d'utilisation commerciale 4.0 International. 


\title{
Le français au Portugal
}

\author{
De la langue si peu « Étrangère » à la difficile normalisation. \\ Considérations et perspectives
}

José Domingues de Almeida

1 Entre les 2èmes Assises du Français au Portugal et celles qui eurent lieu à l'Université de Porto en 2010, un long chemin nous semble avoir été parcouru, et dans le bon sens. Nous nous trouvions alors dans une bien moins bonne posture et l'avenir de l'enseignement efficace de la langue française n'était guère assuré. La déprime et le découragement étaient alors les traits dominants de l'état d'esprit des acteurs de la cause du français, à commencer par les enseignants, dont les effectifs vieillissaient, pataugeaient dans des pratiques didactiques routinières, ou bien se faisaient les apôtres isolés, - mais convaincus, voire militants -, de la promotion de notre langue pour contrecarrer une baisse sensible de classes ayant opté pour le français.

En 2010, des engagements ont été pris par tous les intervenants directs et sur le terrain en vue d'une plus large et meilleure présence du français au Portugal. Si tout n'a pas été mis en place, ou n'aurait peut-être pas pu l'être, des actions concrètes et stratégiques ont quand même été menées qui ont produit des résultats palpables et satisfaisants : une mise à jour didactique s'opère qui passe par de nombreuses initiatives, notamment la rénovation des manuels; le nombre de classes avec option français se stabilise, voire augmente, de façon surprenante et inattendue dans certains établissements scolaires, notamment autour des grands centres urbains du littoral; l'allergie, somme toute irrationnelle, que causait il y a peu le français dans différents milieux a fait place à une redécouverte et à un nouveau constat d'utilité et d'efficace communicationnelles du français, considéré désormais comme un plus dans les formations de tout bord.

La banalisation de l'enseignement-apprentissage du castillan, - que la crise espagnole est venue dévaluer sur le marché des langues - ; la crise sévère au Portugal, qui a acculé à l'émigration, notamment en français, toute une génération de jeunes qualifiés, contraints de revenir en urgence à un usage non élitiste, non prestigieux, mais purement « actionnel et communicationnel » $d u$ français, en situation non plus exolingue, mais en contexte d'immersion forcée, souvent sur le tas; mais aussi une desserte renouvelée de produits culturels et symboliques en français (cinéma, chanson, littérature, etc.) ont contribué à ce 
qui pourrait très bien s'avérer un redémarrage du français, à une autre époque, dans un cadre différent, face à une demande et des publics changeants.

En effet, le rayonnement du français n'a pas toujours été linéaire ou uniforme. Si sa projection et son usage internationaux étaient assuré par l'impact cosmopolite des mouvements et des idées politiques, mais aussi littéraires, elle a cessé de l'être dès que l'anglais s'est imposé comme langue diplomatique et du commerce international à partir de la première guerre mondiale, il y a près de cent ans, alors que le français véhiculait toujours, et en concurrence, de nombreux produits culturels, et que la France s'affichait sur une scène internationale plus limitée et coloniale. Comme le rappelle Paul-Marie Coûteaux, le rayonnement de la langue française, dès la prépondérance anglo-saxonne sur la communication internationale, est avant tout fonction de la capacité des Francophones à susciter des produits culturels et des concepts accrocheurs, comme on l'a vu dans les années 60 et 70 avec le succès du cinéma, du théâtre, du roman et de la critique en français (Coûteaux, 2006).

5 À cet égard, le domaine des produits cosmétiques qui, eux, ne sont pas vraiment "culturels ", - même s'ils réfèrent à des images symboliques -, est très parlant et révélateur. La permanence de la langue française sur les étiquettes et dans les noms de produits assure une authenticité et une fiabilité que l'anglais ne garantit pas à lui seul.

De même, du point de vue terminologique, les emprunts circonstanciels au français demeurent assez fréquents, surtout dans la presse écrite, où ils sont signalés en italique, et marquent, - comme nous le montrions dans un précédent apport (Almeida, 2008) -, la fréquence et la qualité des emprunts au français de la part des plus jeunes locuteurs portugais trahissent un phénomène de mutation psycho- et sociolinguistique qu'il y aurait lieu d'approfondir, statistiques à l'appui. Elles attestent la pertinence symptomatique d'un désaveu et d'un désamour face à la langue française et au rayonnement de sa culture, et des mots pour l'exprimer.

7 De fait, les emprunts, fréquents naguère, et marques d'une posture précieuse et élitiste inscrite dans le discours, se font plus rares chez ces jeunes générations d'apprenants. Des expressions comme noblesse oblige, tout court, à la rigueur, enfant terrible, à la carte, soi disant, à vol d'oiseau, protégé, en passant, malgré lui, parti pris, tête-à-tête, négligé, crème de la crème, entourage [féminin le plus souvent, et à acception politique], rentrée [plus politique que scolaire], etc. demeurent courantes dans la presse écrite, les éditoriaux ou les articles d'opinion, mais ne sont plus compris à l'oral que par une minorité de locuteurs portugais, tandis que les prosaïques c'est fini, c'est la vie, comme ci, comme ça semblent avoir fait l'objet d'une assimilation que l'on retrouve dans les substantifs cache-col, cache-pot, passe-partout, mise (en plis), soutien(-gorge) qui ont, par ailleurs, perdu leur graphie française, et que nuance et habitué résistent assez bien.

8 D'autres exemples attestent une résistance, une imposition pratique ou une connotation culturelle des emprunts nettement perçus comme français: bricolage, guichet, chauffage (féminin), roulement (équipe), ménage (à trois), nécessaire (mala), volte-face, châssis, régie, pivot, plateau (ces trois derniers dans leur acception audiovisuelle), pot-pourri, chance, sommier, dossier, manicure, pédicure etc., dont la graphie n'est plus astreinte à la forme originelle, et encore moins à la prononciation française.

9 Mais les jeunes générations portugaises hésitent à attribuer certains termes courants empruntés au français, et les perçoivent d'abord dans leur accointance ou affinité anglaise : souvenir (tourisme), affair[e] (amoureuse), partenaire / partner (sexuel), plafond 
(teto salarial), déjà vu (du fait de son usage médiatique américain et dont la graphie est souvent simplifiée). Et n'a-t-on pas entendu dans une émission de télévérité fait divers (feitsdaivəz) par un concurrent d'une vingtaine d'années?

Elles hésitent moins, par contre, à opter pour un équivalent anglais, ou différencient subtilement les acceptions : atelier / workshop, équipe / team, tournée / tour, pot-pourri / medley. Fait troublant, elles ne perçoivent pas l'origine française de certains termes liés à des activités où, d'instinct, elles s'imaginent que l'anglais domine d'office : rappel dans les nouvelles modalités sportives dites radicales. Et, à graphie égale, elles privilégient spontanément la prononciation anglaise.

11 En quelque sorte, il s'agirait de pouvoir à nouveau associer l'essor de l'usage international du français à une production culturelle, conceptuelle, artistique, mais aussi économique, issue à présent des vastes espaces francophones, notamment postcoloniaux. Or, un renouveau timide, mais durable, se fait jour depuis quelque temps: deux prix Nobel décernés à peu d'années d'intervalle à deux romanciers francophones (l'un plus hexagonal et l'autre plus littérature-monde), le maintien d'une écriture allophone prisée en français, un cinéma en langue français (tous registres confondus) connaissant un regain de présences dans nos salles et nos écrans, notamment depuis le succès de Le fabuleux destin d'Amélie Poulain (2001), et la distinction aussi bien des films que des comédiens de langue française, notamment à Hollywood. Mêmes espoirs pour la chanson en langue française qui, si elle n'est pas suffisamment diffusée, n'en connaît pas moins des réussites internationales fulgurantes et interculturelles, telles Stromae (Sadaka, 2014 : 6-7), et dont les paroliers font preuve d'une capacité d'invention et d'innovation.

12 Nous osons croire que le français pourrait tenter par là même la chance d'une normalisation communicationnelle chez nous, mais aussi ailleurs, c'est-à-dire devenir une langue utile, efficace et liée aux domaines professionnels porteurs d'avenir: les technologies nouvelles, les activités médicales, le tourisme de masse ou alternatif, les industries culturelles, etc. (Hagège, 2006; Dufour, 2006). Cette gageüre ne serait pas inédite. Que l'on considère, à cet égard, l'impressionnante vitalité de l'allemand dans notre enseignement supérieur alors qu'il est pratiquement absent du secondaire.

13 Ce fait s'explique par l'important relai parallèle du Goethe Institut chez nous, mais aussi par l'association immédiate de cet idiome aux succès et à la fiabilité technologiques de l'industrie allemande. Les entreprises allemandes l'ont bien saisi, n'hésitent pas à exiger une connaissance de l'allemand à l'embauche, et introduisent des slogans publicitaires dans cette langue, incompréhensibles pour la majorité des consommateurs, mais qui s'avèrent un plus et révèlent une attitude décomplexée par rapport à la langue; une posture que la diplomatie allemande suit, elle aussi.

14 On peut regretter que les entreprises françaises installées chez nous ne commencent à exprimer le besoin de l'usage du français que depuis quelque temps. Mais là aussi, les choses bougent dans le bon sens. Il suffirait en fait de persévérer dans une coopération active et intelligente entre la diplomatie linguistique et les acteurs de l'enseignementapprentissage du français sur le terrain, notamment dans l'enseignement supérieur universitaire et polytechnique.

En fait, notre langue se verrait dès lors affranchie du statut purement ornemental ou hyper-prestigieux dans lequel elle s'est vautrée dans nos sociétés depuis le xIx ${ }^{e}$ siècle, un empan qui a assuré son rayonnement, mais a également et paradoxalement concouru à la situation bloquée que l'on a connue ces dernières années, et que nous essayons tant bien 
que mal de palier à présent. L'histoire de l'italien nous montre combien une belle et prestigieuse langue du passé peut très bien ne plus rien peser sur le marché des langues, pour que l'on s'inquiète très concrètement de ce que « le poids » de la langue française à l'échelle globale soit garanti, dans l'acception que Louis-Jean Calvet donne à cette mesure 1 .

Cela suppose une desserte diversifiée de l'enseignement-apprentissage de la langue française ; une langue qu'il faut définitivement acter de « langue étrangère » au Portugal, et dont l'approche doit se normaliser. Concrètement, ceci veut dire que les publics-cibles, ainsi que le profil de nos élèves et étudiants auront irrémédiablement changé; ce que reflète l'adaptation de nos programmes, tant secondaire que supérieur, aux descripteurs de Cadre Européen Commun de Référence pour les Langues (2001).

Aussi, le fait que l'enseignement-apprentissage du français, même à l'Université, puisse commencer au niveau du descripteur A1 doit-il être dédramatisé, même s'il cet apprentissage s'avère bien souvent problématique et implique de nouvelles approches didactiques. Il s'agit simplement d'une approche nouvelle et forcément exigeante, mais motivante, du français que l'on sait ne plus servir au seul embellissement de la personnalité ou de la formation (la cerise sur le gâteau), mais que l'on perçoit plutôt comme un outil utile et performant, ainsi qu'une plus-value curriculaire dans le marché du travail actuel (un ingrédient du gâteau).

Cette nouvelle approche se traduit par la demande plus fréquente aujourd'hui de cours en formation continue, intensifs ou à objectif spécifique immédiatement liés à un intérêt professionnel, notamment dans le tourisme, mais aussi dans l'ensemble des activités économiques, où le français est redevenu une compétence requise, comme le prouvent les annonces d'emploi de nos journaux.

Ce qui ressort de ce que nous avançons ici, c'est que l'efficace de l'action en faveur du français ne peut être que le résultat de synergies aux objectifs et aux acteurs bien ciblés. Rappelons les engagements pris lors des premières Assises de Porto :

1. L'identification et le soutien des expériences de relance du français précoce dans le pays en les divulguant et en leur procurant un suivi didactique et logistique extensible à d'autres expériences et contextes décentralisés d'apprentissage sur tout le territoire ;

2. L'élaboration d'une présentation promotionnelle argumentaire interactive et facilement accessible (diaporama) contenant plusieurs diapositives référentielles de l'univers français et francophone sous forme de module ou de «produit " susceptible de circuler dans les écoles et de procurer une image attrayante et utile de la langue française (personnalités, monuments, chansons, produits, technologie, culture, paysages,...) en vue d'une approche affective et utilitaire du FLE ;

3. L'implication et l'engagement institutionnels des entreprises françaises et francophones installées au Portugal dans le sens d'une approche promotionnelle et d'une plus-value professionnelle du français, notamment à l'embauche et dans la formation continue ;

4. La mise en place d'un réseau de groupe de pression pro-français (langue) en mettant à profit les contacts et les associés de toutes nos associations, à même de s'engager, d'agir et de réagir de façon visible en faveur du rayonnement du français au Portugal ;

5. L'envoi dans les écoles et aux enseignants de 25.000 exemplaires d'un dépliant argumentaire promotionnel en faveur de l'apprentissage du français à tous les niveaux d'enseignement (Santos et al., 2011: 28).

20 De ces différentes mesures tout à fait réalistes, nous dirons que plusieurs ont été appliquées avec le succès que l'on sait, - et l'APEF y est pour beaucoup, comme l'a reconnu 
l'Académie Française en lui décernant le Prix Hervé Deluen. Mais d'autres attendent toujours : le français précoce n'a pas connu de suite, faute d'engagement local, alors que les parents d'élèves y sont largement favorables; les entreprises rechignent toujours à s'afficher "francophones", même si les choses bougent et un outil didactique complet, transversal et mis à jour est toujours demandé par les enseignants, indépendamment des sites didactiques FLE et didacticiels lancés dernièrement par la France.

Mais les autres objectifs ont effectivement abouti à des résultats concrets et palpables sur le terrain ; ceux qu'il nous est permis d'analyser avec optimisme aujourd'hui. En somme, l'enjeu du maintien du français comme langue de communication internationale enseignée et apprise chez nous passe par un processus de normalisation de son statut. Il s'agirait de faire l'exercice difficile, mais, - on s'en aperçoit, « possible » -, de garder le prestige tout en rejetant l'hypostase de la langue; de ne rien perdre des attaches culturelles, tout en les élargissant aux larges espaces francophones, de la "francosphère" (Wolton, 2006: 53), acteurs et objets de la mondialisation; de ne pas perdre de vue le rayonnement, tout en ne tombant pas dans le piège de la mission. Bref, de donner toutes ses chances à la communication. Pour le Portugal, un tel parcours reviendra à accepter la réalité et à encourager l'innovation, le dynamisme et la créativité.

\section{BIBLIOGRAPHIE}

ALMEIDA, José Domingues de (2008). « Que reste-t-il de nos amours? Quelques réflexions sur le statut de la langue française au Portugal aujourd'hui en guise de mise au point et de stratégie didactique », Çédille, no 4, pp. 33-43, <URL : http://webpages.ull.es/users/cedille/cuatro/ almeida.pdf >. [disponible le 29/05/2015]. CONSEIL DE L'EUROPE (2001). Cadre Européen Commun de Référence pour les Langues : apprendre, enseigner, évaluer. Paris : Didier.

CALVET, Louis-Jean - «Le poids des langues », Observatoire Européen du Plurilinguisme. [disponible le 15/12/2004] <URL : http://www.portalingua.info/fr/poids-des-langues/ >.

CALVET, Louis-Jean (1999). Pour une écologie des langues du monde. Paris : Plon.

COÛTEAUX, Paul-Marie (2006). Être et parler français. Paris : Perrin.

DUFOUR, Christian (2006). Le défi français. Montréal : Septentrion.

HAGÈGE, Claude (2006). Combat pour le français. Au nom de la diversité des langues et des cultures. Paris : Odile Jacob.

SADAKA, Edmond (2014). « Stromae : alors il chante », Le Français dans le Monde, no 393, mai-juin, pp. 6-7.

SANTOS, Ana Clara et al. (2011). «Propositions d'engagement », in Ana Clara SANTos, Ana Paula Coutinho, José ALMEIDA, Fátima oUTEIRINHo, Maria DE JESUS CABRAL (orgs.) État des lieux du français au Portugal : observatoire, acteurs \& stratégies de relance, pp. 6-7 [disponible le 12/06/2016] <URL : http://ler.letras.up.pt/uploads/ficheiros/8573.pdf>. 
WOLTon, Dominique (2006). Demain la francophonie. Paris : Flammarion.

\section{NOTES}

1. Cf. CALVET, Louis-Jean - « Le poids des langues », Observatoire Européen du Plurilinguisme. On consultera avec profit, à ce sujet, le travail statistique publié sur http://www.portalingua.info/ fr/poids-des-langues/ [consulté le 15/12/2004].

\section{RÉSUMÉS}

Il s'agira de dresser un bilan générique et systématisé, - tant rétrospectif, perspectif que prospectif -, des mutations dans la perception, le rayonnement et les enjeux de l'enseignementapprentissage de la langue française chez nous; une langue qui n'a, dans le passé, jamais vraiment été « étrangère ", et qui ne parvient toujours pas à trouver un statut normalisé dans la desserte linguistique et le contexte portugais.

We will propose a generic and systematic balance sheet - retrospective, prospective and perspective in the changes in perception, prestige and challenges of teaching and learning of the French language in Portugal; a language that has, in the past, never really been "foreign" and that cannot find yet a normal status in the Portuguese linguistic offer and context

\section{INDEX}

Mots-clés : français, enseignement, apprentissage, Portugal, politique linguistique Keywords : French, teaching, learning, Portugal, linguistic policy

\section{AUTEUR}

\section{JOSÉ DOMINGUES DE ALMEIDA}

Universidade UP - APEF - ILC Margarida Losa

jalmeida[at]letras.up.pt 